\title{
Final focus designs for crab waist colliders
}

\author{
A. Bogomyagkov, ${ }^{1,2, *}$ E. Levichev, ${ }^{1,3}$ and P. Piminov ${ }^{1}$ \\ ${ }^{1}$ Budker Institute of Nuclear Physics SB RAS, Novosibirsk 630090, Russia \\ ${ }^{2}$ Novosibirsk State University, Novosibirsk 630090, Russia \\ ${ }^{3}$ Novosibirsk State Technical University, Novosibirsk 630073, Russia \\ (Received 22 June 2016; published 28 December 2016)
}

\begin{abstract}
The crab waist collision scheme promises significant luminosity gain. The successful upgrade of the $D A \Phi N E$ collider proved the principle of crab waist collision and increased luminosity 3 times. Therefore, several new projects try to implement the scheme. The paper reviews interaction region designs with the crab waist collision scheme for already existent collider DA $\Phi N E$ and SuperKEKB, presently undergoing commissioning, for the projects of SuperB in Italy, CTau in Novosibirsk and FCC-ee at CERN.
\end{abstract}

DOI: 10.1103/PhysRevAccelBeams.19.121005

\section{INTRODUCTION}

Invention of the crab waist collision scheme promises an increase of luminosity by several orders of magnitude for the specially designed collider with respect to conventional. A successful test of the scheme at the existent Italian lepton collider DA $\Phi N E$ increased luminosity 3 times from $1.5 \times 10^{32} \mathrm{~cm}^{-2} \mathrm{~s}^{-1}$ to $4.5 \times 10^{32} \mathrm{~cm}^{-2} \mathrm{~s}^{-1}$ and proved the principle of crab waist. The moderate luminosity gain is due to the limited possibility to implement all of the necessary modifications. Hence, the projects of the new circular colliders exploit the crab waist interaction scheme. However, several designs experienced dynamic aperture degradation trying to implement crab waist sextupoles, e.g. SuperKEKB. In this review we briefly depict the crab waist concept, describe optical designs of interaction regions of the already existent collider DA $\Phi N E$, SuperKEKB, presently undergoing commissioning, the projects of SuperB in Italy, CTau in Novosibirsk and FCC-ee at CERN, discuss the problem of dynamic aperture degradation in several designs and propose an explanation of the effect.

\section{CRAB WAIST COLLISION SCHEME}

Raimondi proposed crab waist collision scheme in 2006 [1]. In [2], Zobov gave a thorough explanation of the scheme. Here we briefly repeat his reasoning for the purpose of clarity and integrity. Three founding steps are at the heart of the crab waist collision scheme. In order to understand these steps, we need expressions for luminosity, vertical and horizontal tune shifts $[3,4]$ :

\footnotetext{
*A.V.Bogomyagkov@inp.nsk.su

Published by the American Physical Society under the terms of the Creative Commons Attribution 3.0 License. Further distribution of this work must maintain attribution to the author(s) and the published article's title, journal citation, and DOI.
}

$L \propto \frac{N \xi_{y}}{\beta_{y}^{*}}, \quad \xi_{y} \propto \frac{N \beta_{y}^{*}}{\sigma_{x}^{*} \sigma_{y}^{*} \sqrt{1+\varphi^{2}}}, \quad \xi_{x} \propto \frac{N \beta_{x}^{*}}{\sigma_{x}^{* 2}\left(1+\varphi^{2}\right)}$

where $N$ is bunch population, $\beta_{x}^{*}$ and $\beta_{y}^{*}$ are horizontal and vertical beta functions at the interaction point (IP), $\sigma_{x}^{*}, \sigma_{y}^{*}$ and $\sigma_{z}$ are horizontal, vertical and longitudinal beam sizes respectfully; using $\theta$ as a full crossing angle, the Piwinski [5] angle is

$$
\varphi=\frac{\sigma_{z}}{\sigma_{x}^{*}} \tan \left(\frac{\theta}{2}\right)
$$

The first step is the large Piwinski angle, which requires long bunches, small horizontal emittance, and a large crossing angle. Hence, $1+\varphi^{2} \approx \varphi^{2}$ and $\sigma_{x}^{*}$ vanishes from the expressions (1). This step reduces vertical tune shift and the size of interaction area (yellow in Fig. 1). Therefore, one desiring to keep vertical tune shift unchanged increases bunch population and gains in luminosity. The second step is reduction of the vertical beta function to half length of the interaction region but not the bunch length. This again makes vertical tune shift smaller and luminosity larger. The third is introduction of the crab sextupoles at the proper phase advances from IP: $\Delta \mu_{x}=\pi \cdot m, \Delta \mu_{y}=\pi / 2 \cdot(2 n+1)$. The sextupoles rotate the position of the vertical beta function waist along the axis of the opposite beam (Fig. 2), and suppress betatron and synchrobetatron resonances [6-9].

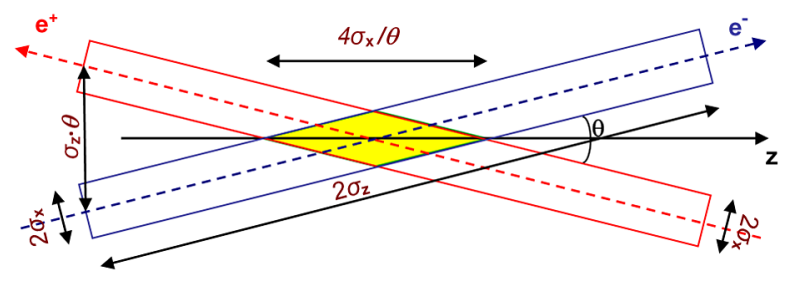

FIG. 1. Layout of the crossing angle collision. 


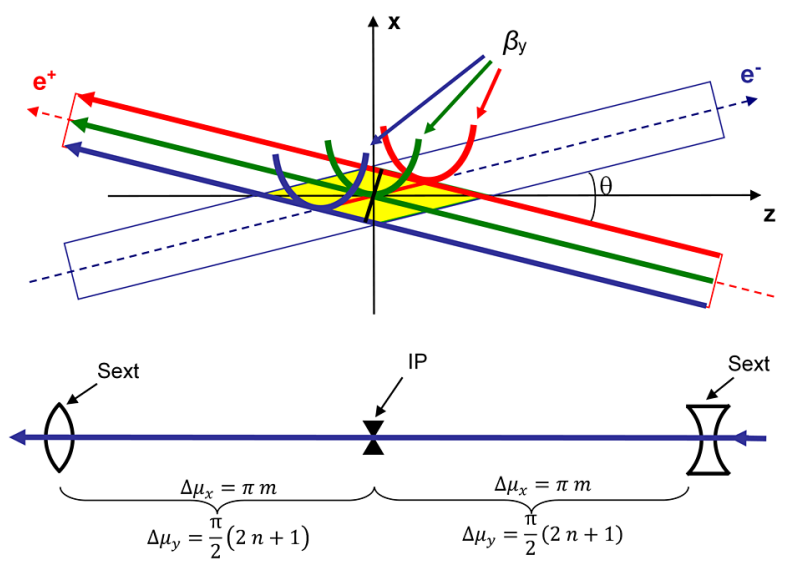

FIG. 2. Crab waist collision scheme.

The integrated strength of the crab sextupoles at the place with vertical $\beta_{y}$ and horizontal $\beta_{x}$ beta functions is

$$
K 2 L= \pm \frac{1}{\theta \beta_{y}^{*} \beta_{y}} \sqrt{\frac{\beta_{x}^{*}}{\beta_{x}}} .
$$

The crab sextupoles cancel each other's second order geometrical aberrations because of proper phase advances and appropriate sign of the field gradient promising no dynamic aperture degradation. So, the whole requirements of the crab waist are: (i) crossing angle, (ii) bunch length, horizontal size and crossing angle should provide large Piwinski angle, (iii) vertical beta function comparable with the size of the interaction area, (iv) sextupoles with proper strength and phase advance from IP.

These requirements are the sources of the difficulties in crab waist implementation: (i) small vertical beta function and desire to minimize beta functions in final quadrupoles, despite the crossing angle, might require double aperture quadrupoles with high gradient; (ii) strong final quadrupoles with large beta function are the source of large chromaticity, and need local chromaticity correction sections; (iii) chromaticity correction sections and final focus quadrupoles will produce large nonlinear chromaticity limiting energy acceptance of the ring; (iv) small horizontal emittance increases chromaticity of the whole ring, and raises the strength of the sextupoles correcting it, and as a result abates dynamic aperture; (v) crab sextupoles require special phase advances from IP and beta functions to reduce the strength of sextupoles, which could be difficult or impossible in the upgrade of the already operating collider; (vi) interference between crab sextupoles and other nonlinear elements of the interaction region might limit dynamic aperture.

\section{NONLINEAR DETUNING}

For comparison of different interaction regions, we will introduce chromaticity produced by final defocusing quadrupoles (from both sides of IP, and final quadrupole could consist of several quadrupoles):

$$
\mu_{y}^{\prime}=\frac{1}{2} \sum_{i} K 1 L_{i} \beta_{i, y}
$$

where $K 1 L_{i}$ is integrated strength of the $i$ th quadrupole, $\beta_{i, y}$ is vertical beta function in the center of the $i$ th quadrupole. The detuning coefficient of the vertical plane $\left(\alpha_{y y}\right)$ with respect to action $J_{y}$,

$$
\Delta \nu_{y}=\alpha_{y y} J_{y}+\alpha_{y x} J_{x}
$$

is the simplest characteristic describing nonlinear properties of the lattice $[10,11]$. It is not an unequivocal attribute: even if detuning (5) is small, higher orders might reduce dynamic aperture. We will consider third order nonlinearities; therefore, the first order detuning allows comparison of different lattices. Since nonlinear effects are much stronger in the vertical plane, we will omit estimations of the horizontal detuning.

Assuming that final focus (FF) quadrupole changes sign of Twiss functions $\alpha_{y}$ we derive the quadrupole integrated strength $K 1 L\left[\mathrm{~m}^{-1}\right]$ :

$$
K 1 L=-\frac{2}{L^{*}+L_{q} / 2},
$$

where $L_{q}$ is quadrupole length, $L^{*}$ is the distance from the IP to the face of the quadrupole. Now we estimate chromaticity as

$$
\mu_{y}^{\prime}=-\frac{L^{*}+L_{q} / 2}{\beta_{y}^{*}} .
$$

From the Hamiltonian of the kinematic term

$$
H=\frac{\left(P_{x}^{2}+P_{y}^{2}\right)^{2}}{8},
$$

we decipher the detuning coefficient for the drift between the FF quadrupoles:

$$
\alpha_{y y}^{k}=\frac{3}{16 \pi} \int \frac{1+\alpha_{y}^{2}}{\beta_{y}^{2}} d s \approx \frac{3}{16 \pi} \frac{L^{*}+L_{q} / 2}{\beta_{y}^{* 2}} .
$$

The Hamiltonian of the fringe field of the FF quadrupole is

$$
H=K_{1}^{\prime} \frac{P_{x} x y^{2}-P_{y} x^{2} y}{4}-K 1^{\prime \prime} \frac{\left(x^{4}-y^{4}\right)}{48},
$$

and we obtain 
TABLE I. Parameters of DA $\Phi$ NE and SuperKEKB.

\begin{tabular}{|c|c|c|c|}
\hline \multirow[b]{2}{*}{ Run or ring } & \multirow{2}{*}{$\frac{\text { DA } \Phi N E}{\text { SIDDHARTA }}$} & \multicolumn{2}{|c|}{ SuperKEKB } \\
\hline & & LER & HER \\
\hline Energy, GeV & 0.51 & 4 & 7.007 \\
\hline Circumference, $\mathrm{m}$ & 97.69 & \multicolumn{2}{|c|}{3016.315} \\
\hline$\varepsilon_{x} / \varepsilon_{y}, \mathrm{~nm} / \mathrm{pm}$ & $250 / 750$ & $3.2 / 8.64$ & $4.6 / 12.9$ \\
\hline$\beta_{x}^{*} / \beta_{y}^{*}, \mathrm{~mm}$ & $250 / 9.3$ & $32 / 0.27$ & $25 / 0.3$ \\
\hline Crossing angle, mrad & 50 & \multicolumn{2}{|c|}{83} \\
\hline$\sigma_{z}, \mathrm{~mm}$ & 17 & 6 & 5 \\
\hline Piwinski angle $\varphi$ & 1.7 & 25 & 19 \\
\hline $\begin{array}{c}\text { Beam current } \\
e^{-} / e^{+}, \mathrm{A}\end{array}$ & $2.45 / 1.4$ & 3.6 & 2.6 \\
\hline $\begin{array}{l}\text { Beam beam tune } \\
\text { shift } \xi_{y}\end{array}$ & 0.03 & 0.088 & 0.08 \\
\hline$\mu_{y}^{\prime}$ & -61 & -5400 & -5400 \\
\hline$\alpha_{y y}^{k}$ & 694 & $1.8 \times 10^{6}$ & $1.8 \times 10^{6}$ \\
\hline$\alpha_{y y}^{f}$ & 218 & $9.8 \times 10^{6}$ & $9.8 \times 10^{6}$ \\
\hline$\alpha_{y y}^{s}$ & & $-7 \times 10^{5}$ & $-7 \times 10^{5}$ \\
\hline \multirow{2}{*}{$\begin{array}{l}\text { Luminosity, } \\
\mathrm{cm}^{-2} \mathrm{~s}^{-1}\end{array}$} & Achieved & \multicolumn{2}{|c|}{ Design } \\
\hline & $4.5 \times 10^{32}$ & \multicolumn{2}{|c|}{$8 \times 10^{35}$} \\
\hline
\end{tabular}

$$
\begin{aligned}
\alpha_{y y}^{f}= & \frac{1}{32 \pi} \int K 1^{\prime \prime} \beta_{y}^{2} d s \approx-\frac{1}{4 \pi} \frac{K 1 \cdot L^{* 3}}{\beta_{y}^{* 2}} \\
\approx & \frac{1}{2 \pi} \frac{L^{* 3}}{L_{q}\left(L^{*}+L_{q} / 2\right) \beta_{y}^{* 2}} .
\end{aligned}
$$

The $-I$ pair of sextupoles $[11,12]$ gives

$$
\alpha_{y y}^{s} \approx-\frac{1}{16 \pi}\left(K 2 L_{s}\right)^{2} L_{s} \beta_{s, y}^{2},
$$

where $K 2$ is sextupole strength $\left[\mathrm{m}^{-3}\right], L_{s}$ is sextupole length, $\beta_{s, y}$ is vertical beta functions at the sextupole position.

\section{PRESENT COLLIDERS: DA $\Phi$ NE AND SUPERKEKB}

\section{A. DA $\Phi \mathbf{N E}$}

DA $\Phi N E$ is an electron-positron collider with central mass energy of $1.02 \mathrm{GeV}$ ( $\Phi$ resonance) delivering luminosity since 2000 [2]. The staff upgraded the machine to implement the crab waist scheme in 2007. The changes included 2 times larger crossing angle, 26\% smaller horizontal emittance, almost 2 times smaller vertical and horizontal beta functions, and 50\% smaller bunch length. Reduction of the bunch length was not intentional and happened because of continuous work on impedance reduction. Constraints of the already working machine did not allow achieving extreme parameters; nevertheless, they doubled the Piwinski angle from 0.8 to 1.7 (Table I) and increased luminosity 3 times [13]. Moderate IP beta

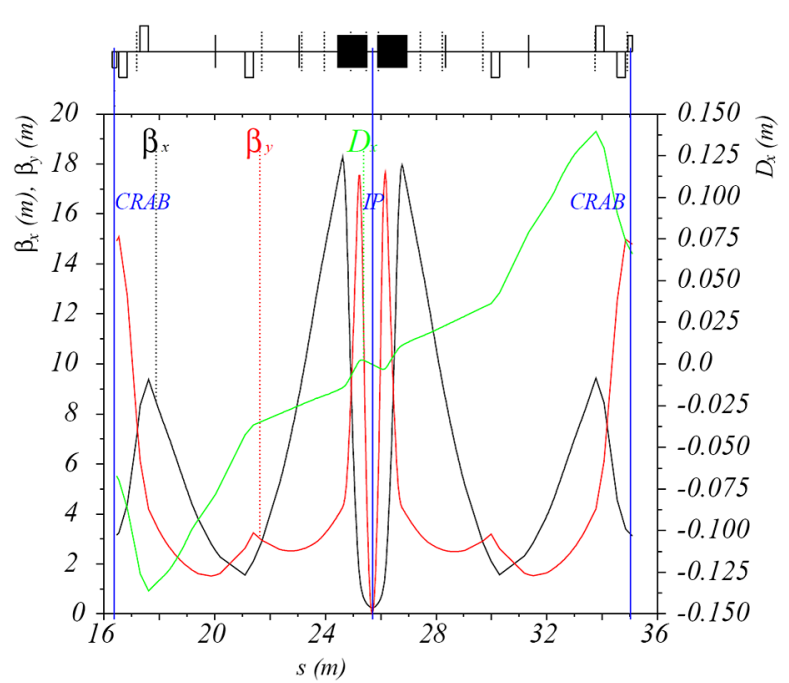

FIG. 3. Optical functions of DA $\Phi N E$ interaction region with crab waist sextupoles.

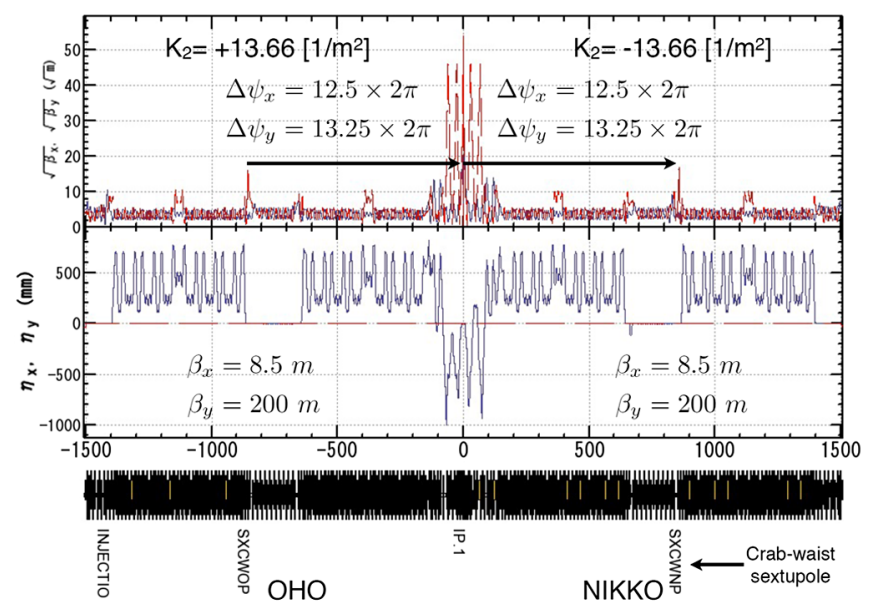

FIG. 4. Optical functions of SuperKEKB LER interaction region with crab waist sextupoles.

functions and small beta functions in final quadrupoles do not require separate chromaticity correction sections, and sextupoles of the ring correct the whole chromaticity. Figure 3 shows optical functions of the DA $\Phi$ NE interaction region.

\section{B. SuperKEKB}

SuperKEKB [14-16] is an upgrade of the KEKB B-factory [14] in the state of beam commissioning [17] with the goal to increase luminosity 40 times to $0.8 \times 10^{36} \mathrm{~cm}^{-2} \mathrm{~s}^{-1}$ (Table I). The upgrade followed the steps of the crab collision scheme and, because of very small beam sizes at IP, received the name of nano-beam. Figures 4 and 5 show optical functions of the interaction region for low (LER) and high (HER) energy rings. The crab sextupoles are installed before horizontal and vertical 


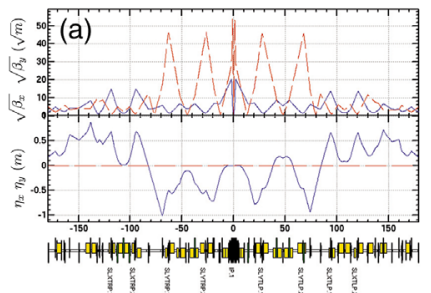

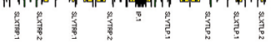

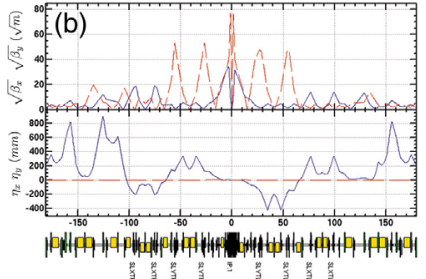

FIG. 5. Optical functions of SuperKEKB interaction region: (a) LER, (b) HER.

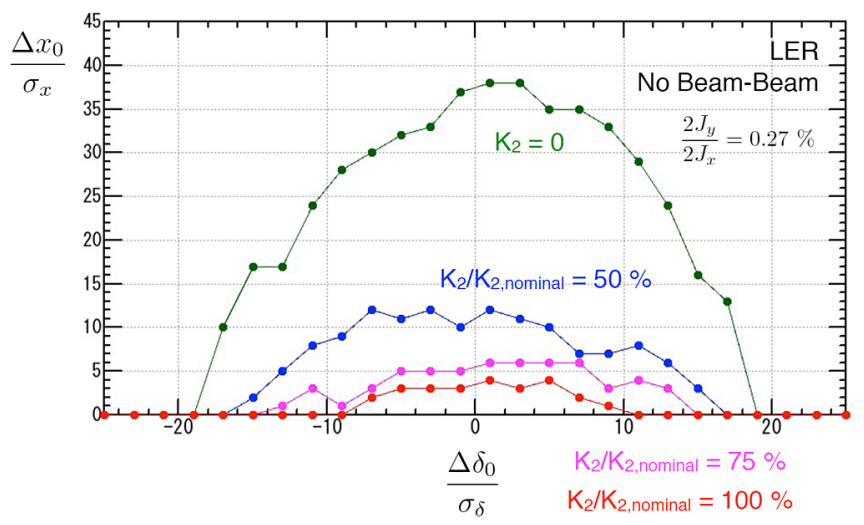

FIG. 6. Dynamic aperture for LER SuperKEKB with different crab sextupole strength.

chromaticity sections, rather far from the IP at $\mu_{x}=12.5 \times$ $2 \pi$ and $\mu_{y}=13.25 \times 2 \pi$. The interplay of the crab sextupole, the nonlinear fringe of final quadrupoles, and the kinematic term in the IP drift reduces dynamic aperture drastically [18,19] (Fig. 6). The staff did not find a solution to regain dynamic aperture; therefore, they planned to work without crab sextupoles.

TABLE II. Parameters of SuperB.

\begin{tabular}{lcc}
\hline \hline & \multicolumn{2}{c}{ SuperB } \\
\cline { 2 - 3 } Run or ring & LER & HER \\
\hline Energy, GeV & 4.18 & 6.7 \\
Circumference, m & 1258.4 \\
$\varepsilon_{x} / \varepsilon_{y}, \mathrm{~nm} / \mathrm{pm}$ & $2.46 / 6.15$ & $2 / 5$ \\
$\beta_{x}^{*} / \beta_{y}^{*}, \mathrm{~mm}$ & $32 / 0.205$ & $26 / 0.253$ \\
Crossing angle, mrad & \multicolumn{2}{c}{66} \\
$\sigma_{z}, \mathrm{~mm}$ & 5 & 5 \\
Piwinski angle $\varphi$ & 19 & 23 \\
Beam current, A & 2.4 & 1.9 \\
Beam beam tune shift $\xi_{y}$ & 0.097 & 0.097 \\
$\mu_{y}^{\prime}$ & -1068 & -1056 \\
$\alpha_{y y}^{k}$ & $1 \times 10^{6}$ & $1 \times 10^{6}$ \\
$\alpha_{y y}^{f}$ & $2.8 \times 10^{5}$ & $2.8 \times 10^{5}$ \\
$\alpha_{y y}^{s}$ & $-5.4 \times 10^{6}$ & $-5.4 \times 10^{6}$ \\
Luminosity, $\mathrm{cm}^{-2} \mathrm{~s}^{-1}$ & \multicolumn{2}{c}{$1 \times 10^{36}$} \\
\hline \hline
\end{tabular}

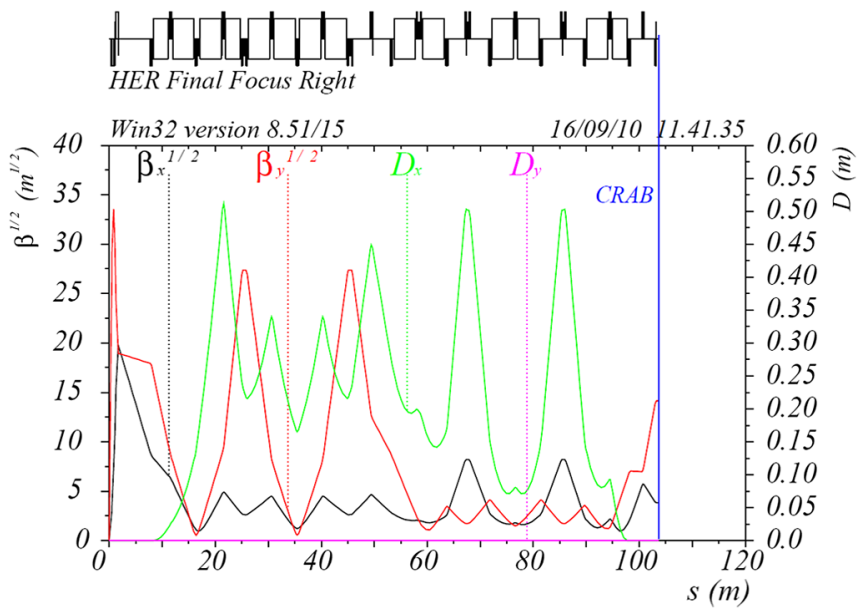

FIG. 7. Optical functions of SuperB HER interaction region with crab waist sextupoles.

\section{PROJECTS BASED ON CRAB WAIST}

\section{A. SuperB}

SuperB $[20,21]$ is an Italian project (canceled by the government) of asymmetric $b$ factory employing the crab waist collision scheme to achieve luminosity of $1 \times 10^{36} \mathrm{~cm}^{-2} \mathrm{~s}^{-1}$ (Table II). The optics of the interaction region includes separate vertical and horizontal chromaticity correction sections followed by crab sextupole (Fig. 7). Again, dynamic aperture shrinks under influence of crab sextupoles (Fig. 8), but it is satisfactory.

\section{B. CTau}

Super Charm-Tau Factory is a project of the electronpositron collider in the Budker Institute of Nuclear Physics (Novosibirsk, Russia) [22]. The designed center mass energy range of operation is from 2 to $5 \mathrm{GeV}$ with luminosity reaching $1 \times 10^{35} \mathrm{~cm}^{-2} \mathrm{~s}^{-1}$ (Table III). It also relies on the crab waist collision scheme. China proposed a similar project HIEPA [23].

Interaction region optics, similar to SuperB, consists of separate chromaticity correction sections (sextupoles Y1 and $\mathrm{Y} 3, \mathrm{X} 1$ and $\mathrm{X} 3$ ) and crab sextupole (Fig. 9). The optics also includes additional sextupoles $\mathrm{Y} 2$ and $\mathrm{Y} 4, \mathrm{X} 2$ and $\mathrm{X} 4$ to correct reduction of dynamic aperture due to finite length

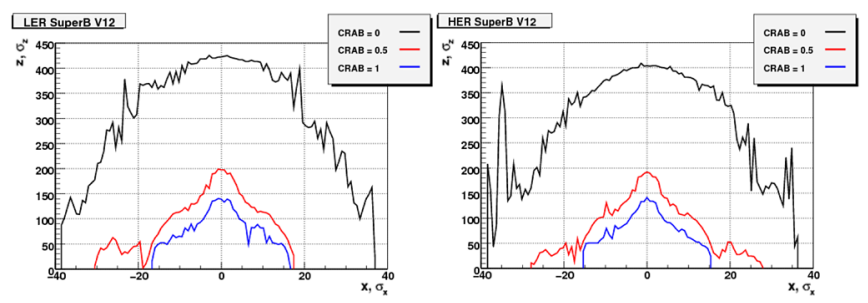

FIG. 8. On momentum dynamic aperture of SuperB LER and HER: black line—crab sextupoles are off, red and blue-crab sextupoles strength of $50 \%$ and $100 \%$ of nominal respectfully. 
TABLE III. Parameters of CTau in Novosibirsk.

\begin{tabular}{|c|c|c|c|c|}
\hline \multirow[b]{2}{*}{ Energy, GeV } & \multicolumn{2}{|c|}{ CTau } & \multirow[b]{2}{*}{2} & \multirow[b]{2}{*}{2.5} \\
\hline & 1 & 1.5 & & \\
\hline Circumference, $\mathrm{m}$ & \multicolumn{4}{|c|}{813.4} \\
\hline$\varepsilon_{x} / \varepsilon_{y}, \mathrm{~nm} / \mathrm{pm}$ & \multicolumn{4}{|c|}{$8 / 40$} \\
\hline$\beta_{x}^{*} / \beta_{y}^{*}, \mathrm{~mm}$ & \multicolumn{4}{|c|}{$40 / 0.8$} \\
\hline $\begin{array}{l}\text { Crossing angle, } \\
\text { mrad }\end{array}$ & \multicolumn{4}{|c|}{60} \\
\hline$\sigma_{z}, \mathrm{~mm}$ & 16.5 & 11 & 10 & 10 \\
\hline Piwinski angle $\varphi$ & 27 & 19 & 17 & 17 \\
\hline Beam current, A & \multicolumn{4}{|c|}{1.65} \\
\hline $\begin{array}{l}\text { Beam beam tune } \\
\text { shift } \xi_{y}\end{array}$ & 0.15 & 0.15 & 0.12 & 0.1 \\
\hline$\mu_{y}^{\prime}$ & \multicolumn{4}{|c|}{-697} \\
\hline$\alpha_{y y}^{k}$ & \multicolumn{4}{|c|}{$1.3 \times 10^{5}$} \\
\hline$\alpha_{y y}^{f}$ & \multicolumn{4}{|c|}{$7.7 \times 10^{5}$} \\
\hline$\alpha_{y y}^{s}$ & \multicolumn{4}{|c|}{$-7.2 \times 10^{5}$} \\
\hline $\begin{array}{l}\text { Luminosity, } \\
\mathrm{cm}^{-2} \mathrm{~s}^{-1}\end{array}$ & $0.6 \times 10^{35}$ & $0.9 \times 10^{35}$ & $1 \times 10^{35}$ & $1 \times 10^{35}$ \\
\hline
\end{tabular}

of the main sextupoles [12], and sextupoles X5, X6, X7 help to correct nonlinear chromaticity $[24,25]$.

\section{FCC-ee}

The future circular collider is a project at CERN of the next accelerator after LHC [26,27]. The ultimate goal is a $100 \mathrm{~km}$ proton-proton machine with $100 \mathrm{TeV}$ central mass energy. The first possible step is the $e^{+} e^{-}$factory-FCC-ee with central mass energy range from 80 to $350 \mathrm{GeV}$ and two IPs (Table IV).

Minimization of synchrotron radiation background towards the detector and the length of IR tunnel are important requirements; therefore, IR is asymmetric, i.e. with lower bending for the incoming beam and stronger bending for outgoing beam. Two teams developed different IR optics [28,29]. The first variant (Fig. 10) does not have a

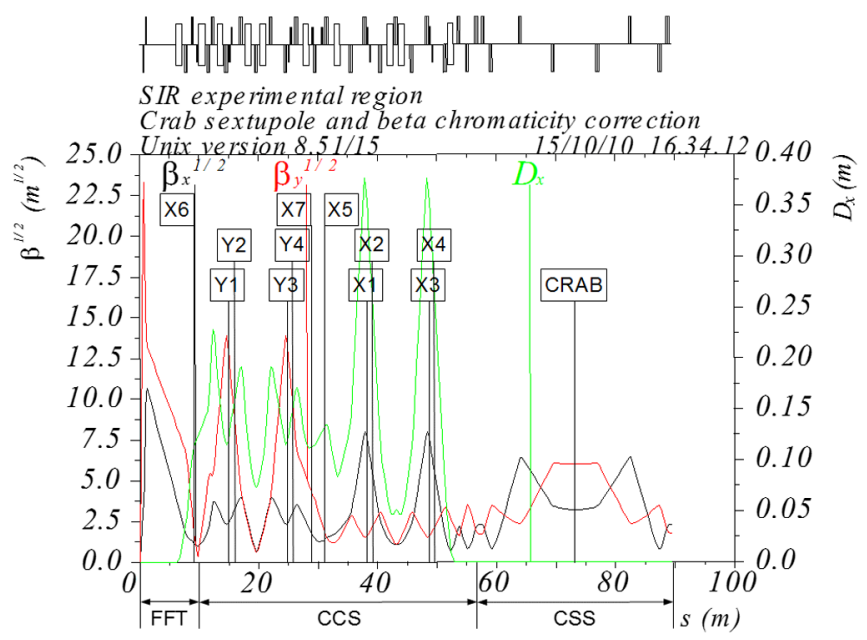

FIG. 9. Optical functions of the CTau interaction region.
TABLE IV. Parameters of FCC-ee.

\begin{tabular}{|c|c|c|c|c|}
\hline \multirow[b]{2}{*}{ Experiment } & \multicolumn{2}{|c|}{ FCC-ee } & \multirow[b]{2}{*}{$\mathrm{H}$} & \multirow[b]{2}{*}{$\mathrm{tt}$} \\
\hline & $\mathrm{Z}$ & W & & \\
\hline Energy, GeV & 45 & 80 & 120 & 175 \\
\hline Circumference, $\mathrm{m}$ & \multicolumn{4}{|c|}{$100 \times 10^{3}$} \\
\hline$\varepsilon_{x} / \varepsilon_{y}, \mathrm{~nm} / \mathrm{pm}$ & $0.14 / 1$ & $0.44 / 2$ & $1 / 2$ & $2.1 / 4.3$ \\
\hline$\beta_{x}^{*} / \beta_{y}^{*}, \mathrm{~mm}$ & \multicolumn{4}{|c|}{$500 / 1$} \\
\hline $\begin{array}{l}\text { Crossing angle, } \\
\text { mrad }\end{array}$ & \multicolumn{4}{|c|}{30} \\
\hline$\sigma_{z}, \mathrm{~mm}$ & 5.9 & 9.1 & 8.2 & 6.6 \\
\hline $\begin{array}{l}\text { Piwinski } \\
\quad \text { angle } \varphi\end{array}$ & 11 & 9 & 6 & 3 \\
\hline Beam current, A & 1.4 & 1.4 & 0.3 & 0.06 \\
\hline $\begin{array}{l}\text { Beam beam } \\
\text { tune shift } \xi_{y}\end{array}$ & 0.175 & 0.187 & 0.16 & 0.08 \\
\hline$\mu_{y}^{\prime}$ & \multicolumn{4}{|c|}{-2805} \\
\hline$\alpha_{y y}^{k}$ & \multicolumn{4}{|c|}{$4.5 \times 10^{5}$} \\
\hline$\alpha_{y y}^{f}$ & \multicolumn{4}{|c|}{$1.9 \times 10^{5}$} \\
\hline$\alpha_{y y}^{s}$ & \multicolumn{4}{|c|}{$-1.2 \times 10^{7}$} \\
\hline $\begin{array}{l}\text { Luminosity, } \\
\qquad \mathrm{cm}^{-2} \mathrm{~s}^{-1}\end{array}$ & $211 \times 10^{34}$ & $36 \times 10^{34}$ & $9 \times 10^{34}$ & $1.3 \times 10^{34}$ \\
\hline
\end{tabular}

horizontal chromaticity section because of geometrical constraints. The second sextupole of the $-I$ pair performs two functions: it cancels geometric aberrations of the first sextupole and, because dispersion is zero, it plays a role of crab sextupole. Individual $-I$ pairs of arc sextupoles correct nonlinear chromaticity and dynamic aperture (Fig. 11).

The second variant (Fig. 12) employs a separate horizontal chromaticity corrections section and additional sextupoles as in the CTau project. The arc sextupoles constitute two families. Dynamic aperture is comparable with the variant one (Fig. 13). Switching-on crab sextupoles degrades dynamic aperture for interaction region variant 2 (Fig. 14).

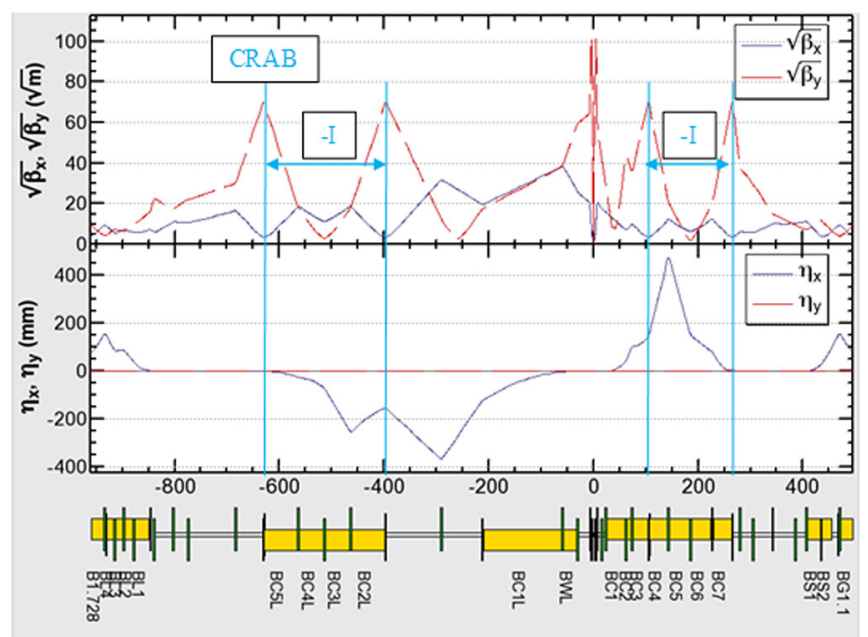

FIG. 10. Optical functions of FCC-ee interaction region variant 1 . 


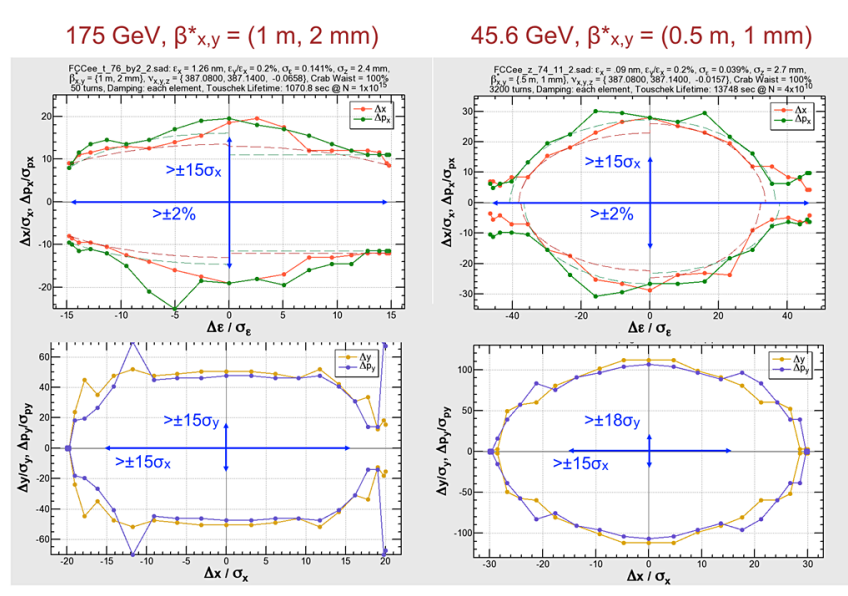

FIG. 11. Dynamic aperture for FCC-ee interaction region variant 1 .

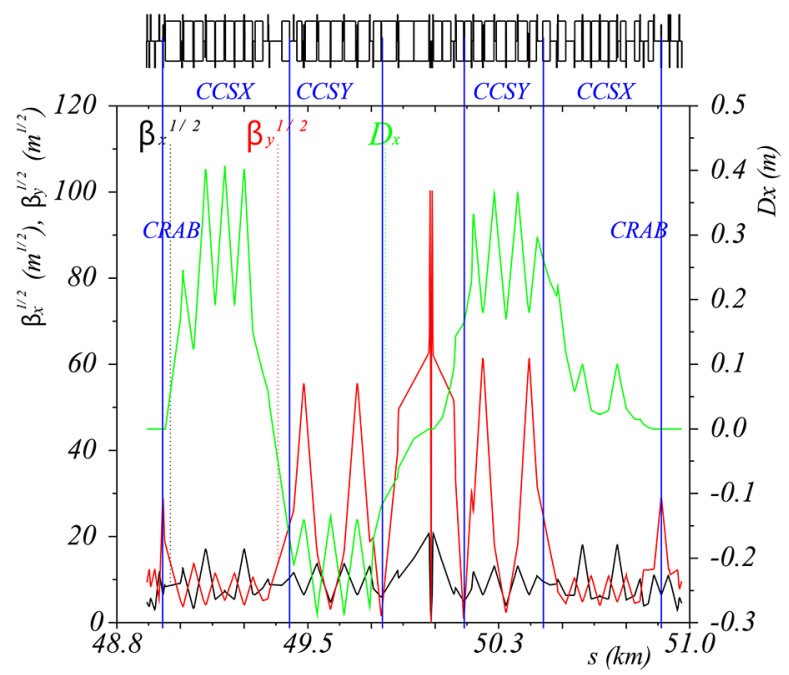

FIG. 12. Optical functions of FCC-ee interaction region variant 2 .

\section{DISCUSSION}

$D A \Phi N E$ is the only collider among the reviewed projects which does not report significant dynamic aperture loss from the crab sextupoles. Observing detuning coefficients, we notice that SuperKEKB has the highest coefficients for kinematic term and for quadrupole fringe (Table V). The authors of [12] described the effect of sextupole length and its compensation, therefore we do not discuss the large detuning coefficient from sextupole pairs. The source of dynamic aperture reduction is then interference of crab

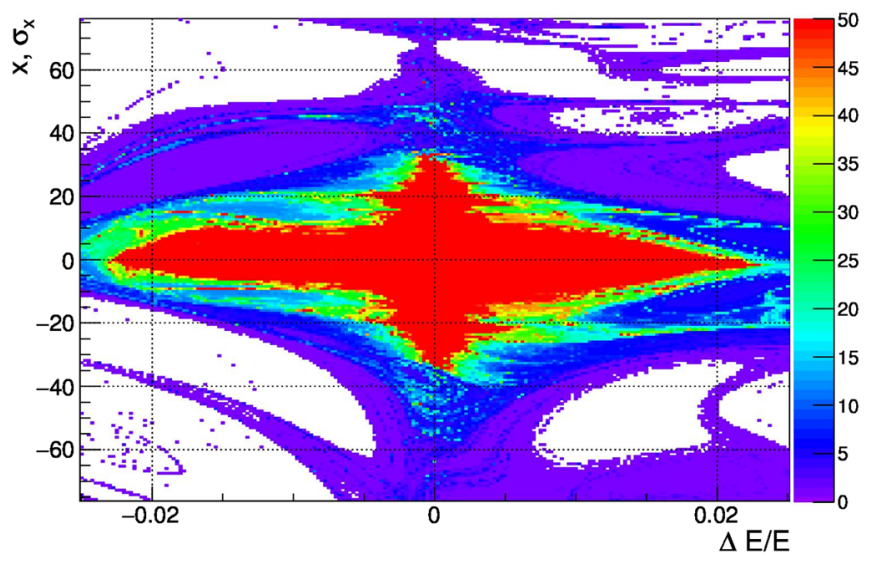

FIG. 13. Dynamic aperture for the FCC-ee interaction region variant 2 (50 turns, without damping, crab sextupole is off, rf is on).

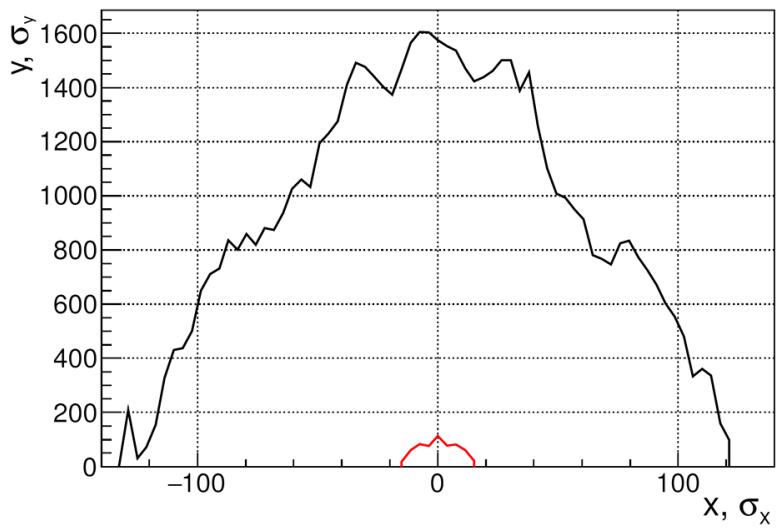

FIG. 14. Dynamic aperture degradation for FCC-ee interaction region variant 2 , black is with crab sextupole OFF, red with crab sextupole $\mathrm{ON}$, includes only nonlinearities of the interaction region, on momentum particles.

sextupoles with nonlinearities of kinematic terms and quadrupole fringes. To understand the nature of the dynamic aperture loss, we calculated the transfer map for a simple symmetrical case of thin crab sextupoles with strength $\pm K 2 L\left[\mathrm{~m}^{-2}\right]$, thin final quadrupoles placed at the middle of the real thick magnets with fringes described by $K 1 L\left[\mathrm{~m}^{-1}\right]$ and $K 1\left[\mathrm{~m}^{-1}\right]$, two drifts of the length $L^{*}$ (from quadrupole to IP) with kinematic terms (Fig. 15). Coordinates after the second crab sextupole depend on initial $x_{0}, y_{0}$ at the entrance of the first sextupole as

$$
\begin{aligned}
x= & x_{0}-x_{0}^{4} \frac{K 1 \cdot K 1 L \cdot L^{* 2}}{\theta \beta_{y}^{*} \beta_{y}} \sqrt{\frac{\beta_{x}^{*}}{\beta_{x}}}+y_{0}^{4} \frac{L^{*}\left(1+2 \cdot K 1 \cdot K 1 L \cdot L^{* 3}\right)}{2 \theta \beta_{y}^{* 2} \beta_{y}^{2}} \sqrt{\frac{\beta_{x}}{\beta_{x}^{*}}} \\
& -x_{0}^{2} y_{0}^{2} \frac{L^{*}\left[\left(1+2 \cdot K 1 \cdot K 1 L \cdot L^{* 3}\right) \beta_{x}+6 \cdot K 1 \cdot K 1 L \cdot L^{*} \beta_{x}^{*} \beta_{y}^{*} \beta_{y}\right]}{2 \theta \beta_{y}^{* 2} \beta_{y}^{2} \sqrt{\beta_{x}^{*} \beta_{x}}},
\end{aligned}
$$


TABLE V. Comparison of detuning coefficients [expressions (5), (9), (11), and (13)].

\begin{tabular}{lrccr}
\hline \hline & \multicolumn{1}{c}{$\mu_{y}^{\prime}$} & $\alpha_{y y}^{k}\left[\mathrm{~m}^{-1}\right]$ & $\alpha_{y y}^{f}\left[\mathrm{~m}^{-1}\right]$ & \multicolumn{1}{c}{$\alpha_{y y}^{s}\left[\mathrm{~m}^{-1}\right]$} \\
\hline DAФNE & -61 & 694 & 218 & \\
SuperKEKB & -5400 & $1.8 \times 10^{6}$ & $9.8 \times 10^{6}$ & $-7 \times 10^{5}$ \\
SuperB & -1060 & $1 \times 10^{6}$ & $2.8 \times 10^{5}$ & $-5.4 \times 10^{6}$ \\
CTau & -700 & $1.3 \times 10^{5}$ & $7.7 \times 10^{5}$ & $-7.2 \times 10^{5}$ \\
FCC-ee v.2 & -2800 & $4.5 \times 10^{5}$ & $1.9 \times 10^{5}$ & $-1.2 \times 10^{7}$ \\
\hline \hline
\end{tabular}

$p_{x}=-x_{0}^{3} \frac{2}{3} K 1 \cdot K 1 L \frac{\beta_{x}^{* 2}}{\beta_{x}^{2}}-x_{0} y_{0}^{2} \frac{2 \cdot K 1 \cdot K 1 L \cdot L^{* 2} \beta_{x}^{*}}{\beta_{y}^{*} \beta_{y} \beta_{x}}$,

$y=-y_{0}-x_{0}^{3} y_{0} \frac{2 \cdot K 1 \cdot K 1 L}{\theta}\left[\frac{L^{* 2}}{\beta_{y}^{*} \beta_{y}} \sqrt{\frac{\beta_{x}^{*}}{\beta_{x}}}+\left(\frac{\beta_{x}^{*}}{\beta_{x}}\right)^{\frac{3}{2}}\right]$,

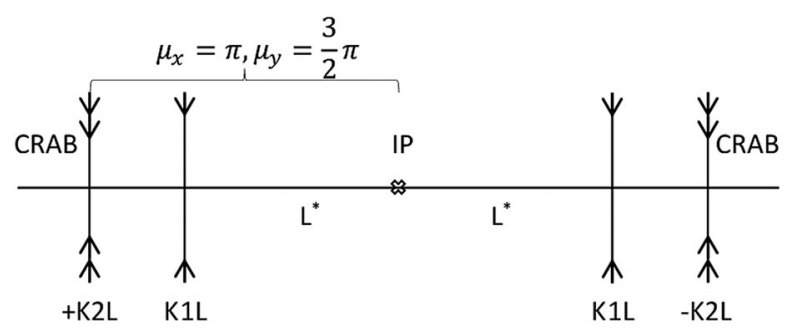

FIG. 15. Layout of the simple interaction region.

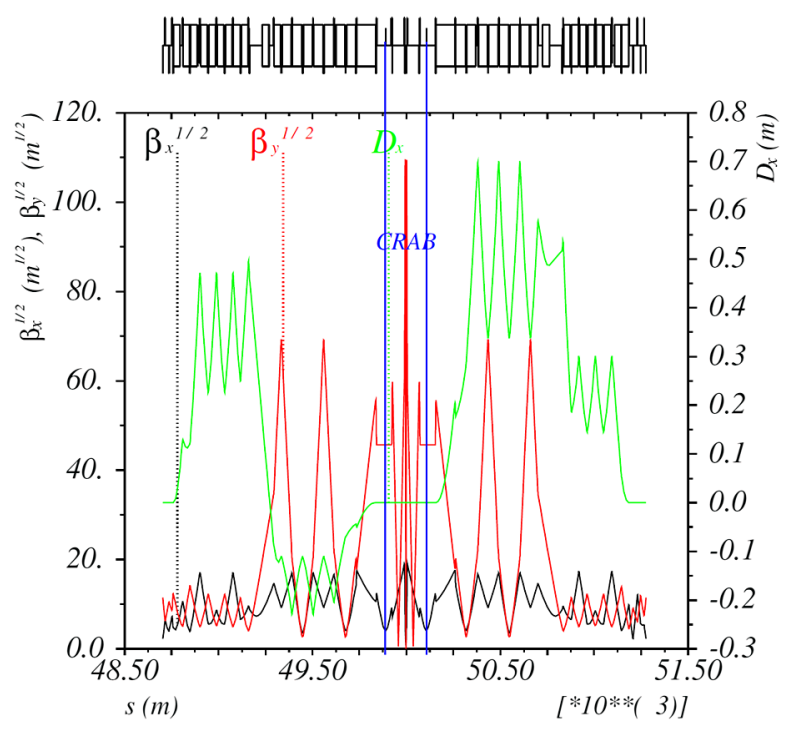

FIG. 16. Optical functions of FCC-ee interaction region variant 3 .
TABLE VI. The changes between FCC-ee versions 2 and 3.

\begin{tabular}{lcc}
\hline \hline & FCC-ee v. 2 & FCC-ee v.3 \\
\hline$\beta_{x}[\mathrm{~m}]$ & 42 & 16 \\
$\beta_{y}[\mathrm{~m}]$ & 835 & 2086 \\
$L^{*}[\mathrm{~m}]$ & 2 & 2.9 \\
$L_{q}[\mathrm{~m}]$ & 3.6 & 1.8 \\
\hline \hline
\end{tabular}

$$
\begin{aligned}
p_{y}= & y_{0}^{3} \frac{L^{*}\left(3+2 \cdot K 1 \cdot K 1 L \cdot L^{* 3}\right)}{3 \beta_{y}^{* 2} \beta_{y}^{2}} \\
& +x_{0}^{2} y_{0} \frac{2 \cdot K 1 \cdot K 1 L \cdot L^{* 2} \beta_{x}^{*}}{\beta_{y}^{*} \beta_{y} \beta_{x}}
\end{aligned}
$$

where we preserved the same notation for beta functions, and we chose $p_{x, 0}=0, p_{y, 0}=0$ for simplicity, and substituted crab sextupole strength (3). We notice that in order to minimize the nonlinear map coefficients one needs to increase $\theta, \beta_{y}, L_{q}$ (then $K 1 \cdot K 1 L$ will decrease) and decrease $L^{*}, \beta_{x}$. In order to check our observations we modified variant 2 of FCC-ee interaction region (Fig. 16). Table VI presents relevant changes between FCC-ee interaction regions version 2 and 3. The plot on Fig. 17 shows dynamic aperture degradation for FCC-ee interaction region variant 3. Comparison with Fig. 14 reveals that dynamic aperture for FCC-ee interaction region variant 3 increased from $20 \sigma_{x} \rightarrow 100 \sigma_{x}, 100 \sigma_{y} \rightarrow 200 \sigma_{y} \quad\left(\sigma_{x}=2.6 \times 10^{-5} \mathrm{~m}\right.$, $\left.\sigma_{y}=5.3 \times 10^{-8} \mathrm{~m}\right)$, supporting our observation that interference of crab sextupoles with kinematic terms and fringes of the final focus quadrupoles is responsible for dynamic aperture degradation. Introducing map notation $U_{i j k l}$ and $V_{\mathrm{ijklm}}$ for vector $z=\left\{x, p_{x}, y, p_{y}\right\}$ $\left(z_{i}=U_{i j k l} z_{j} z_{k} z_{l}+V_{i j k l m} z_{j} z_{k} z_{l} z_{m}\right)$, we compare the largest coefficients for different projects, Tables VII, VIII, and IX, and notice that SuperKEKB has the largest coefficient from the interference of crab sextupoles and quadrupole fringes.

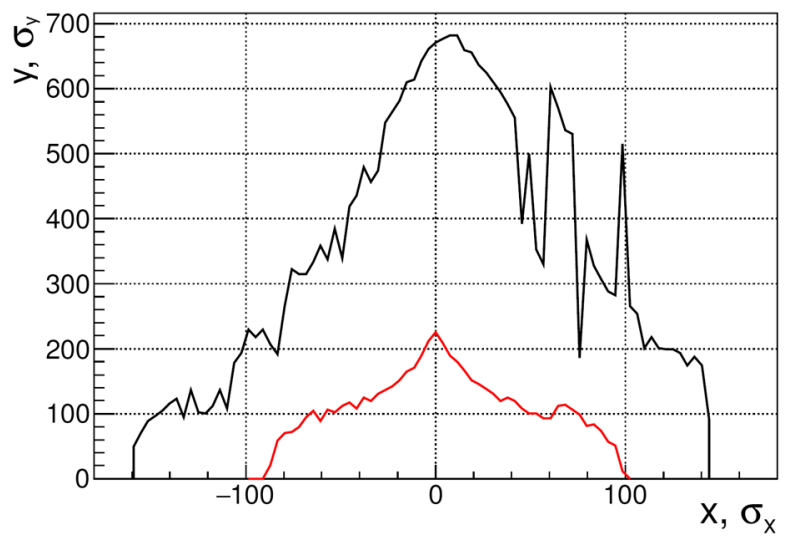

FIG. 17. Dynamic aperture degradation for FCC-ee interaction region variant 3 , black is with crab sextupole OFF, red with crab sextupole $\mathrm{ON}$, includes only nonlinearities of the interaction region, on momentum particles. 
TABLE VII. Comparison of the map coefficients for DA $\Phi$ NE and SuperKEKB.

\begin{tabular}{lcc}
\hline \hline & $\mathrm{DA} \Phi \mathrm{NE}$ & SuperKEKB \\
\hline$V_{11111}, \mathrm{~m}^{-3}$ & $-21 \cdot K 1 \cdot K 1 L=-521$ & $-16.5 \cdot K 1 \cdot K 1 L=-125$ \\
$V_{11133}, \mathrm{~m}^{-3}$ & $-6632-1005 \cdot K 1 \cdot K 1 L=-3 \times 10^{4}$ & $-37036-98641 \cdot K 1 \cdot K 1 L=-7.8 \times 10^{5}$ \\
$V_{13333}, \mathrm{~m}^{-3}$ & $6632+941 \cdot K 1 \cdot K 1 L=3 \times 10^{4}$ & $37036+98591 \cdot K 1 \cdot K 1 L=7.8 \times 10^{5}$ \\
$U_{2111}, \mathrm{~m}^{-3}$ & $-0.005 \cdot K 1 \cdot K 1 L=-0.1$ & $-9.5 \times 10^{-6} \cdot K 1 \cdot K 1 L=-7 \times 10^{-5}$ \\
$U_{2133}, \mathrm{~m}^{-3}$ & $-0.6 \cdot K 1 \cdot K 1 L=-15$ & $-0.2 \cdot K 1 \cdot K 1 L=-1.3$ \\
$V_{31113}, \mathrm{~m}^{-3}$ & $-44 \cdot K 1 \cdot K 1 L=-10^{3}$ & $-33 \cdot K 1 \cdot K 1 L=-250$ \\
$U_{4113}, \mathrm{~m}^{-3}$ & $0.6 \cdot K 1 \cdot K 1 L=15$ & $0.2 \cdot K 1 \cdot K 1 L=1.3$ \\
$U_{4333}, \mathrm{~m}^{-3}$ & $191+9 \cdot K 1 \cdot K 1 L=413$ & $377+335 \cdot K 1 \cdot K 1 L=3 \times 10^{3}$ \\
\hline \hline
\end{tabular}

TABLE VIII. Comparison of the map coefficients for SuperB and CTau.

\begin{tabular}{lcc}
\hline \hline & SuperB & CTau \\
\hline$V_{11111}, \mathrm{~m}^{-3}$ & $-5.5 \cdot K 1 \cdot K 1 L=-28$ & $-15 \cdot K 1 \cdot K 1 L=-470$ \\
$V_{11133}, \mathrm{~m}^{-3}$ & $-67680-42577 \cdot K 1 \cdot K 1 L=-2.9 \times 10^{5}$ & $-75241-51660 \cdot K 1 \cdot K 1 L=-1.7 \times 10^{6}$ \\
$V_{13333}, \mathrm{~m}^{-3}$ & $67680+42561 \cdot K 1 \cdot K 1 L=2.9 \times 10^{5}$ & $75241+51615 \cdot K 1 \cdot K 1 L=1.7 \times 10^{6}$ \\
$U_{2111}, \mathrm{~m}^{-3}$ & $-1 \times 10^{-6} \cdot K 1 \cdot K 1 L=-5.6 \times 10^{-6}$ & $-1 \times 10^{-5} \cdot K 1 \cdot K 1 L=-3.5 \times 10^{-4}$ \\
$U_{2133}, \mathrm{~m}^{-3}$ & $-0.03 \cdot K 1 \cdot K 1 L=-0.13$ & $-0.1 \cdot K 1 \cdot K 1 L=-3.6$ \\
$V_{31113}, \mathrm{~m}^{-3}$ & $-11 \cdot K 1 \cdot K 1 L=-55$ & $-30 \cdot K 1 \cdot K 1 L=-10^{3}$ \\
$U_{4113}, \mathrm{~m}^{-3}$ & $0.03 \cdot K 1 \cdot K 1 L=0.13$ & $0.1 \cdot K 1 \cdot K 1 L=3.6$ \\
$U_{4333}, \mathrm{~m}^{-3}$ & $33+67 \cdot K 1 \cdot K 1 L=664$ & $578+132 \cdot K 1 \cdot K 1 L=4.7 \times 10^{3}$ \\
\hline \hline
\end{tabular}

TABLE IX. Comparison of the map coefficients for FCC-ee variants 2 and 3.

\begin{tabular}{lcc}
\hline \hline & FCC variant 2 & FCC variant 3 \\
\hline$V_{11111}, \mathrm{~m}^{-3}$ & $-63 \cdot K 1 \cdot K 1 L=-2$ & $-41 \cdot K 1 \cdot K 1 L=-4$ \\
$V_{11133}, \mathrm{~m}^{-3}$ & $-833-91554 \cdot K 1 \cdot K 1 L=-4 \times 10^{3}$ & $-82-9158 \cdot K 1 \cdot K 1 L=-1 \times 10^{3}$ \\
$V_{13333}, \mathrm{~m}^{-3}$ & $833+91365 \cdot K 1 \cdot K 1 L=4 \times 10^{3}$ & $82+9035 \cdot K 1 \cdot K 1 L=1 \times 10^{3}$ \\
$U_{2111}, \mathrm{~m}^{-3}$ & $-9 \times 10^{-5} \cdot K 1 \cdot K 1 L=-3 \times 10^{-6}$ & $-7 \times 10^{-4} \cdot K 1 \cdot K 1 L=-7 \times 10^{-5}$ \\
$U_{2133}, \mathrm{~m}^{-3}$ & $-0.4 \cdot K 1 \cdot K 1 L=-0.015$ & $-0.4 \cdot K 1 \cdot K 1 L=-0.04$ \\
$V_{31113}, \mathrm{~m}^{-3}$ & $-126 \cdot K 1 \cdot K 1 L=-4.5$ & $-82 \cdot K 1 \cdot K 1 L=-8.2$ \\
$U_{4113}, \mathrm{~m}^{-3}$ & $0.4 \cdot K 1 \cdot K 1 L=0.015$ & $0.4 \cdot K 1 \cdot K 1 L=0.04$ \\
$U_{4333}, \mathrm{~m}^{-3}$ & $5+199 \cdot K 1 \cdot K 1 L=13$ & $0.9+32 \cdot K 1 \cdot K 1 L=4$ \\
\hline
\end{tabular}

\section{ACKNOWLEDGMENTS}

We express our gratitude to Mikhail Zobov for details about the DA $\Phi$ NE upgrade. This work has been supported by Russian Science Foundation (Project No. 1450-00080).

[1] P. Raimondi, Status of the SuperB effort, in 2nd Workshop on Super B factory, LNF-INFN, Frascati, 2006, http:// www.lnf.infn.it/conference/superb06/talks/raimondi1.ppt.

[2] M. Zobov, New generation electron-positron factories, Phys. Part. Nucl. 42, 782 (2011).

[3] P. Raimondi and M. Zobov, DA $\Phi$ NE Technical Note No. G-58, 2003.
[4] D. Shatilov and M. Zobov, Beam-beam collisions with an arbitrary crossing angle: Analytical tune shifts, tracking algorithm without Lorentz boost, crab-crossing, ICFA Beam Dyn. Newslett. 37, 99 (2005).

[5] A. Piwinski, Satellite resonances due to beam-beam interaction, IEEE Trans. Nucl. Sci. 24, 1408 (1977).

[6] D. V. Pestrikov, Vertical synchrobetatron resonances due to beam-beam interaction with horizontal crossing, Nucl. Instrum. Methods Phys. Res., Sect. A 336, 427 (1993).

[7] P. Raimondi, D. N. Shatilov, and M. Zobov, Beam-beam issues for colliding schemes with large Piwinski angle and crabbed waist, arXiv:physics/0702033.

[8] P. Raimondi, D. Shatilov, and M. Zobov, Suppression of beam-beam resonances in crab waist collisions, Conf. Proc. C0806233 (2008) WEPP045.

[9] D. Shatilov, E. Levichev, E. Simonov, and M. Zobov, Application of frequency map analysis to beam-beam 
effects study in crab waist collision scheme, Phys. Rev. ST Accel. Beams 14, 014001 (2011).

[10] A. V. Bogomyagkov, E. B. Levichev, P. A. Piminov, A. Chance, B. Dalena, J. Payet, R. D. Maria, S. Fartoukh, and M. Giovannozzi, Analysis of the non-linear fringe effects of large aperture triplets for the HL LHC project, in Proceedings of the 4th International Particle Accelerator Conference (IPAC-2013), Shanghai, China, 2013 (JACoW, Shanghai, China, 2013), WEPEA049, http://JACoW.org/IPAC2013/ papers/wepea049.pdf.

[11] A. Bogomyagkov, E. Levichev, and P. Piminov, Nonlinear Perturbations for High Luminosity $e^{+} e^{-}$Collider Interaction Region (IAS HEP Conference, Hong Kong, 2016).

[12] A. Bogomyagkov, S. Glukhov, E. Levichev, and P. Piminov, Effect of the sextupole finite length on dynamic aperture in the collider final focus, arXiv:0909.4872.

[13] M. Zobov et al., Test of Crab-Waist Collisions at DA $\Phi N E$ $\Phi$ Factory, Phys. Rev. Lett. 104, 174801 (2010).

[14] K. Oide, KEKB B-Factory, the luminosity frontier, Prog. Theor. Phys. 122, 69 (2009).

[15] T. Abe et al., Belle II Technical Design Report, arXiv: 1011.0352.

[16] Y. Ohnishi et al., Accelerator design at SuperKEKB, Prog. Theor. Exp. Phys. (2013) 03A011.

[17] Y. Funakoshi et al., Beam commissioning of SuperKEKB, in Proceedings of 7th International Particle Accelerator Conference (IPAC 2016), Busan, Korea (2016), TUOBA01, http://inspirehep.net/record/1469865/files/tuoba01.pdf.

[18] Y. Ohnishi, Optics issues, in 18th KEKB Review KEK, 2014.
[19] Y. Ohnishi, Dynamic aperture optimization in SuperKEKB, in Proceedings of 55th ICFA Advanced Beams Dynamics Workshop on High Luminosity Circular $e^{+} e^{-}$ Colliders Higgs Factory (HF2014), Beijing, China (2015), FRT1B2, http://jacow.org/HF2014/papers/frt1b2.pdf.

[20] M. Bona et al., SuperB: A High-Luminosity Asymmetric $e^{+} e^{-}$Super Flavor Factory. Conceptual Design Report, arXiv:0709.0451.

[21] M. Biagini et al., SuperB progress report, arXiv: 1009.6178.

[22] V. Anashin et al., A project of super C-tau factory in Novosibirsk, conceptual design report (Budker Institute of Nuclear Physics, Novosibirsk, 2011).

[23] Z. Zhou et al., Preliminary concept and key technologies of HIEPA accelerator, in Proceedings of 7 th International Particle Accelerator Conference (IPAC 2016) (2016), THPOR047, http://inspirehep.net/record/1470642/files/ thpor047.pdf.

[24] R. Brinkmann, Report No. DESY M-90-14, 1990.

[25] A. Bogomyagkov, Chromaticity correction of the interaction region (IAS Program on High Energy Physics, Hong Kong, 2016).

[26] M. Benedikt, Future Circular Collider Study: Status and parameter update (2nd FCC week, Rome, 2016).

[27] FCC-ee design study, http://tlep.web.cern.ch.

[28] K. Oide et al., Design of beam optics for the Future Circular Collider $e^{+} e^{-}$collider rings, Phys. Rev. Accel. Beams 19, 111005 (2016).

[29] A. Bogomyagkov, Interaction region optics solutions (2nd FCC week, Rome, 2016). 